\title{
Cerebral Autoregulation Following Traumatic Brain Injury
}

\author{
Gill E. Sviri*,1 and David W. Newell ${ }^{2}$ \\ ${ }^{I}$ Department of Neurological Surgery, Rambam Medical Center, the Technion, Israel institution of Technology Haifa \\ Israel \\ ${ }^{2}$ Department of Neurological Surgery, University of Washington, Seattle, WA, USA. Swedish Neuroscience Institute, \\ Seattle, WA, USA
}

\begin{abstract}
Cerebral pressure autoregulation (AR) is a complex intrinsic control mechanism which maintains a constant cerebral blood flow (CBF). This mechanism was found to be impaired after traumatic brain injury (TBI) and was suggested to be associated with variety of cerebrovacular abnormalities found after injury, as disturb AR might increases the vulnerability of the brain to secondary ischemic insult. Several investigators have found disturb AR response after TBI to be associated with poor outcome and increased mortality, suggesting that impaired AR might reduce the ability of injured brain to preserve an adequate blood flow in the face of hypertensive episodes. Despite the considerable diversity of methodological approaches most studies of cerebral pressure AR in patients with mild or severe TBI have show that autoregulation is often impaired, with a time course that can be quite variable. AR recovery after severe TBI can be delayed and failure to recover during the second week after injury can be found mainly in unfavorable outcome patients.
\end{abstract}

Keywords: Brain injury, autoregulation, blood flow, transcranial doppler, outcome.

\section{INTRODUCTION}

Cerebral autoregulation (AR) is an intrinsic control mechanism which maintains a constant cerebral blood flow (CBF) by constantly changing in the cerebral vascular resistance in response to changing arterial blood pressure (BP) or cerebral perfusion pressure (CPP). This mechanism was found to be impaired after brain injury, even minor $[1,2]$ and was suggested to increase brain tissue vulnerability to secondary ischemic insult resulting for reduction in the BP or elevated ICP [2-5]. In a patient with preserved Cerebral AR, a step CPP decrease would elicit a dilatation of cerebral resistance vessels, which would tend to compensate for CPP decrease. On the other hand, if AR was totally absent, vessels diameter would not change, and CBF would decrease in the same degree that the pressure drop. Several investigators have found impaired AR response after TBI to be associated with poor outcome and increased mortality [2,6-8], as impaired AR reduces the ability of injured brain to preserve an adequate blood flow in the face of hypertensive episodes [24]. This increased vulnerability of injured brain to secondary hemodynamic impairments has increased awareness of the importance of maintaining a perfusion pressure in TBI and a targeted perfusion pressure therapy has progressively gained wide acceptance [1].

\section{METHODS FOR MEASUREMENTS OF CEREBRAL PRESSURE AUTOREGULATION}

Several methods have been suggested for measurements of AR, all involved evaluation of blood flow velocities

*Address correspondence to this author at the Department of Neurosurgery, Rambam (Maimonides) Medical Center, The Technion, Israel Institution of Technology, P.O.B 31096, Haifa, Israel; Tel: 9724 8541840;

Fax: 9724 8543664; E-mail: sviri@u.washington.edu
(BFVs) using transcranial Doppler (TCD) monitoring combined with BP manipulation. Authors have reported diversity in the AR strength that can be found when measured by different methods. At the moment there is no "gold standard" in this matter. In the dynamic cerebral AR measurements the response of cerebral BFVs are been measured in respond to a step blood pressure drop. In the static cerebral AR measurements, the changes in cerebral BFVs are measured after a slow hypertensive challenge. Therefore, the static and the dynamic AR measurements explore two different sides of the autoregulatory process. The dynamic explore the vasodilatation and the hypotensive side and the static the hypertensive side and the vasoconstriction.Furthermore, the vessel tone at the moment when the stimulus is applied may also be responsible for different autoregulation strength in each side of the CBF plateau. Some patients who show an impaired dynamic response to a steep hypotensive challenge may, however, maintain a good response in the steady state, and on the other hand, patients with an effective reaction to steady hypertension may show a very poor response to a rapid drop of blood pressure [9-11].

\section{Dynamic Method for Measurements of AR Strength}

The changes in blood pressure and BFVs of the proximal middle cerebral arteries (MCAs) immediately before and after the thighs cuff release are used to calculate an AR index (ARI) that reflects relative changes in blood flow through the MCA caused by the change in distal cerebral vascular resistance per second, relative to the change in mean arterial blood pressure (MABP). The TCD therefore monitors the relative change in blood flow through the inflow vessels (bilateral MCAs) during the autoregulatory response to a transient drop in blood pressure. A hypothetical curve of the CBF velocity values is created based on the MABP immedi- 
ately prior to the drop and during the 30 seconds thereafter. If there were no autoregulation, the relative changes in $\mathrm{CBF}$ velocity would parallel the changes in MABP and the ARI would be zero. The computer calculates nine other possible responses to the given change in MABP based on a stepwise improvement in the autoregulatory response. The actual response is examined and the "best fit" with the model (the lowest standard error of the mean of the difference between the model and the actual response) is taken as the ARI. The higher the ARI, the more rapid and efficient is the autoregulatory response.

\section{Static Method for Measurements of AR Strength}

In the static AR testing a slow (median duration of the tests: $20 \mathrm{~min}$ ) increase in MAP, elicited by the use of a continuous infusion of phenylephrine or noradrenaline, while continuously recording MAP, intracranial pressure (ICP) and BFVs. Static autoregulation is calculated as the percentage change in cerebral vascular resistance (CVR) in relation to the change in CPP over the entire period of time needed for a MAP increase from baseline to the highest level. An index of static autoregulation (sRoR) was calculated: $s R o R=\% \Delta$ $\mathrm{eCVR} / \% \Delta \mathrm{CPP} \times 100$, with $\% \Delta \mathrm{eCVR}=(\mathrm{eCVR} 2-$ $\mathrm{eCVR} 1) / \mathrm{eCVR} \times 100$, and $\Delta \mathrm{CPP}=(\mathrm{CPP} 2-\mathrm{CPP} 1) / \mathrm{CPP} 1 \times$ 100. This index expresses the change in resistances as a percentage of the complete autoregulatory capacity. If the change in CVR is enough to compensate for the drop in CPP, the sRoR would be $100 \%$, and in the other hand, the absence of a vasoconstrictive response would yield a sRoR of $0 \%$. A value greater than $60 \%$ is considered preserved CA.

\section{DISCUSSION}

\section{AR Impairment in TBI}

The majority of the severe TBI patients experienced impaired AR within the first 48 hours after the injury. Hlatky et al. [4] using the same cuff deflation dynamic AR testing found that only 16 of 122 severe TBI patients had AR index within normal range on day 2 after the injury. Muizelaar et al. [5] using static AR testing found disturbed AR in 15 of 37 measurements done in pediatric patients. This high rate of
AR impairment reported after severe head injury, is not surprising, and of interest is that Junger et al. [12] found absent of AR during the rarely phase in $28 \%$ of 29 patients with minor head injury. In our previously study on time course of AR recovery after sever TBI [13] using dynamic testing we found that during days 3-5 after the injury a larger proportion of severe TBI patients experienced impaired AR response ( $83 \%$ of 36 , Fig. 1), the mean AR index measured reached a lower value of $1.36 \pm 0.24$ and $36 \%$ of 36 patients had poor AR response. Afterward, a gradual improvement was found, however the mean AR response on days 9-11 were still below the normal range and $53 \%$ of 36 patients had impaired AR response at this time period as the majority of them (75\% of 20 ) had poor or absence AR response. Unlike days 1-5 after the injury, during which most of the disturbed AR patients experienced moderately impaired AR, the majority of the patients with impaired AR on days 6-14 had poor or absence AR response. Actually most of the patients with the moderately impaired AR showed a recovery in the end of the first week and beginning of the second week after the injury, whereas patients with poor or absent AR failed to show a recovery at this time period and eventually experienced AR recovery by the end of the second week and beginning of the third week after the injury. Our findings are consisting with observations of Czosnyka et al. [2] who found, using a different methodology, the AR index to continually and gradually deteriorate from day 3 to 8 after the injury, after showing some improvement from between day 1 and 3. Haltky et al. [4] reported that although ARIs were gradually improved by day 10 after the injury the fifth day values were similar to the initial values taken within 12 hour after the injury.

\section{$A R$ and Severity of the Injury}

AR response after TBI is highly associated with the severity of primary and secondary brain damage measured by the type of brain injury (diffuse Vs focal) GCS and intracranial pressure (Fig. 2). Patients with either focal brain injury, higher GCS $(6 \leq)$ or controlled ICP had a tendency towered earlier AR recovery (on days 5-11) and for an overall moderate AR impairment [13]. However, diffuse brain injury, lower GCS (3-5) or elevated ICP were associated with a late

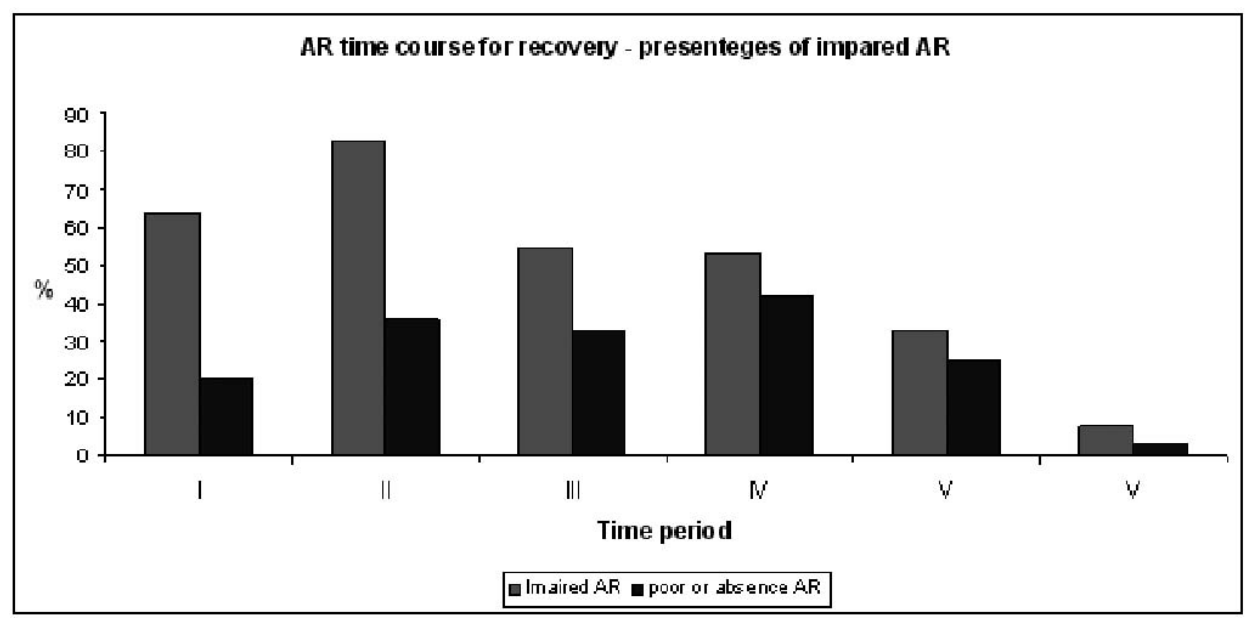

Fig. (1). Proportion of patients with impaired AR response (AR Index: $\geq 1 ;<2.8$ ) and poor AR response after severe TBI (AR index $<1$ ) using dynamic AR measurements and time period after the injury. Period I: within 48 hours from time of injury; period II: days 3-5 after the injury; period III: days 6-8; period IV: days 9-11; period V: days12-14; period VI: days 15-18; period VII: days 19-23. Sviri et al. J Neurosurg, 2009; 111: 695 . 
$\mathbf{A}$

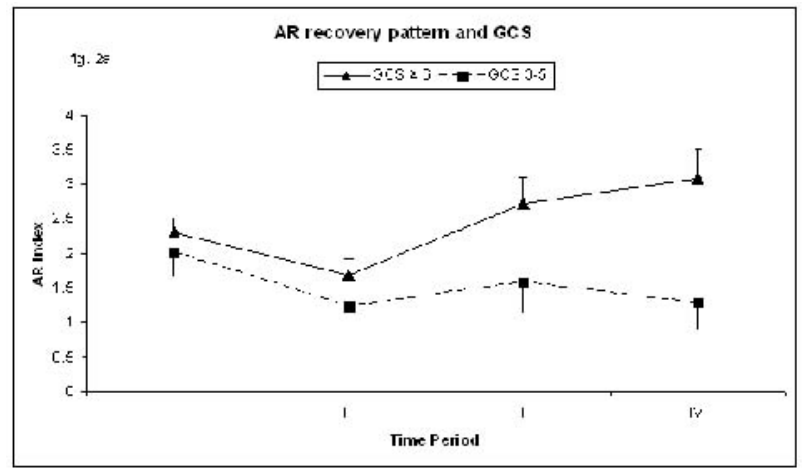

$\rho \div 3.55$
B

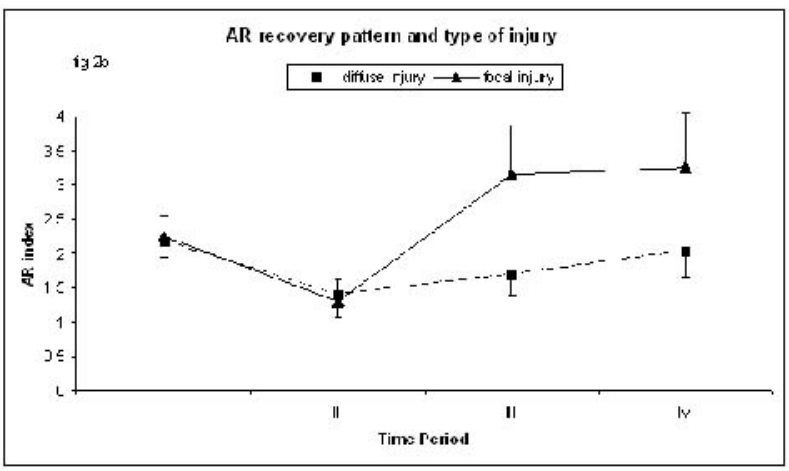

$2 \times 0.0^{\circ}$

C

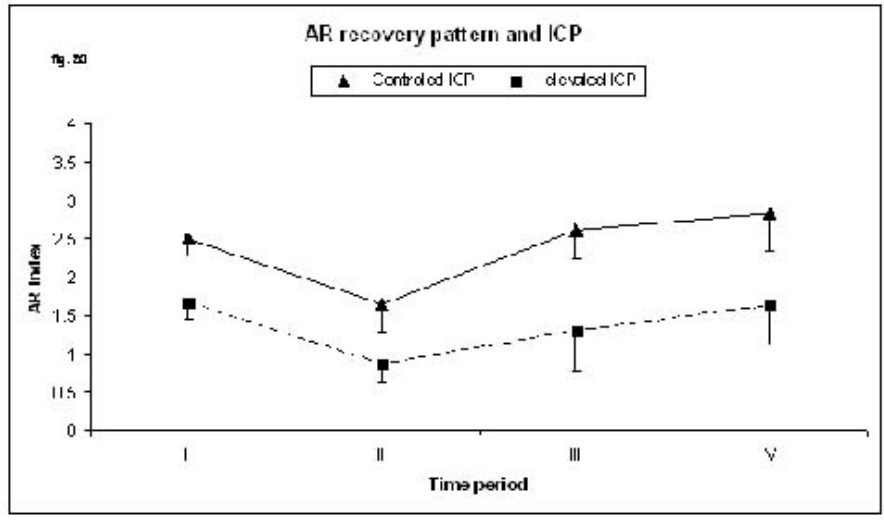

$\xi<0.05$

Fig. (2). Mean ( \pm SEM) AR index value over time periods I-IV as related to Glasgow coma score GCS (A), type of injury (focal Vs diffuse) (B) ICP (C) and 6 months outcome using Glasgow outcome score GOS (D) in 36 patients with severe TBI. $p$ value stands for multivariate ANOVA. Period I: within 48 hours from time of injury; period II: days 3-5 after the injury; period III: days 6-8; period IV: days 9-11. Sviri et al. J Neurosurg, 2009; 111: 695.

AR recovery pattern, occurring in the end of second week after the injury, and many patients experienced prolonged AR "paralysis" (poor or absence AR) over that time period [13].

\section{AR and Outcome}

Many authors have reported on a correlation between impairments of AR and outcome. Paneri et al. [7] evaluated correlation between $\mathrm{AR}$, mortality and outcome in 32 severe TBI and found the ARI to be significantly lower for nonsurvivors compared with survivors $(\mathrm{p}=0.0004)$ with significant correlation between ARI and GOS ( $\mathrm{r}=0.464 ; p=0.011)$. Steiger et al. [8] found correlation between the rate of dynamic AR and GOS and Czosnyka et al. [3] evaluating 187 patients with severe TBI found that patients with unfavorable outcome had significantly $(\mathrm{p}<0.00002)$ lower AR values. Also consistant with our findings Czosnyka et al. [3] found impaired AR to be associated with poor outcome as well as with elevated ICP and lower GCS. In our previously study [13] twenty of 36 patients had favorable outcome six month after the injury. Patients with unfavorable outcome showed tendency for late AR recovery as $75 \%, 65 \%$ and $50 \%$ of them had impaired AR on period III-IV (respectively) compared to $25 \%, 37.5 \%$ and $12.5 \%$ in the favorable outcome patients (Fig. 2). Furthermore, eight of 20 patients (40\%) with unfavorable 6 months outcome had poor AR on period $\mathrm{V}$ compared to one of 16 patients with favorable outcome $(6 \%, p<0.05)$. The AR recovery pattern in the favorable outcome group was significantly different than the unfavorable outcome groups, ( $p=0.007$, Fig. 2).

Whether impaired AR is directly associated with poor outcome depends on other variables not yet defined as profound prolonged impaired AR might increases brain vulnerability to BP insults; AR impairment might be aggravated or prolonged by elevated ICP and pressure support therapy as was addressed by Czosnyka et al. [3] as 'chicken or egg' issue and further study should be done in order to evaluated clinical variables associated with AR impairment.

\section{CONCLUSION}

Cerebral AR is found to be impaired after TBI with prong disturbance that can be found is some patients. After sever TBI, AR impairment can be profound with delayed recovery increasing secondary brain insults resulting from drops in the CPP during the ICU period. This prolongs and profound AR disturbance can be found in patients with an unfavorable outcome. Although different methods are in use 
for a bad side measurements of CAR, currently there is not proven gold standard as the different methods explore contradicted faces of the vasodilatatory response $[9,10]$ Therefore in some of the TBI patients the vasodilatator response can be impaired with intact vasoconstrictive response. Nevertheless, it is believed that the dynamic AR is more relevant in the clinical setting as it is measuring the response to a droop in the BP. Currently, the impact of impaired AR on patients outcome is unclear. Patients with a favorable outcome experienced an earlier time course for recovery. Therefore perfusion pressure management and adequate BP maintenance should be considered in some severe TBI patients for a period of at least two weeks.

\section{REFERENCES}

[1] Brain Trauma Foundation; American Association of Neurological Surgeons; Congress of Neurological Surgeons; Joint Section on Neurotrauma and Critical Care, AANS/CNS. I. Blood pressure and oxygenation. J Neurotrauma 2007; 24: s7-s13.

[2] Czosnyka M, Smielewski P, Kirkpatrick P, Menon DK, Pickard JD. Monitoring of cerebral autoregulation in head-injured patients. Stroke 1996; 27: 1829-34.

[3] Czosnyka M, Smielewski P, Piechnik S, Steiner LA, Pickard JD. Cerebral autoregulation following head injury. J Neurosurg 2001; 95: 756-63.

[4] Hlatky R, Furuya Y, Valadka AB, et al. Dynamic autoregulatory response after severe head injury. J Neurosurg 2002; 97: 1054-61.
[5] Muizelaar JP, Ward JD, Marmarou A, Newlon PG, Wachi A Cerebral blood flow and metabolism in severely head-injured children. Part 2: Autoregulation. J Neurosurg 1989; 71: 72-76.

[6] Czosnyka M, Smielewski P, Kirkpatrick P, Laing RJ, Menon D, Pickard JD. Continuous assessment of the cerebral vasomotor reactivity in head injury. Neurosurgery 1997; 41: 11-17.

[7] Panerai RB, Kerins V, Fan L, Yeoman PM, Hope T, Evans DH. Association between dynamic cerebral autoregulation and mortality in severe head injury. Br J Neurosurg 2004; 18: 471-19.

[8] Steiger HJ, Aaslid R, Stooss R, Seiler RW. Transcranial Doppler monitoring in head injury: relations between type of injury, flow velocities, vasoreactivity, and outcome. Neurosurgery 1994; 34 79-85.

[9] Tiecks FP, Lam AM, Aaslid R, Newell DW. Comparison of static and dynamic cerebral autoregulation measurements. Stroke 1995; 26: 1014-19.

[10] Puppo C, López L, Caragna E, Biestro A. One-minute dynamic cerebral autoregulation in severe head injury patients and its comparison with static autoregulation. A transcranial Doppler study. Neurocrit Care 2008; 8: 344-52.

[11] Newell DW, Aaslid R, Stooss R, Seiler RW, Reulen HJ. Evaluation of hemodynamic responses in head injury patients with transcranial Doppler monitoring. Acta Neurochir (Wien) 1997; 139: 804-17.

[12] Junger EC, Newell DW, Grant GA, Avellino AM, Ghatan S, Douville CM, Lam AM, Aaslid R, Winn HR. Cerebral autoregulation following minor head injury. J Neurosurg 1997; 86: 425-32.

[13] Sviri GE, Aaslid R, Douville CM, Moore A, Newell DW. Time course for autoregulation recovery following severe traumatic brain injury. J Neurosurg 2009; 111: 695-700.

(C) Sviri and Newell; Licensee Bentham Open.

This is an open access article licensed under the terms of the Creative Commons Attribution Non-Commercial License (http://creativecommons.org/licenses/by-nc/3.0/) which permits unrestricted, non-commercial use, distribution and reproduction in any medium, provided the work is properly cited. 